Supplementary Information

\title{
Residual structures and transient long-range interactions of p53 transactivation domain: Assessment of explicit solvent protein force fields
}

\author{
Xiaorong $\mathrm{Liu}^{1}$ and Jianhan Chen ${ }^{1,2 *}$ \\ ${ }^{1}$ Department of Chemistry and ${ }^{2}$ Department of Biochemistry and Molecular Biology, \\ University of Massachusetts Amherst, Amherst, MA 01003, USA \\ *Corresponding Authors: Emails: jianhanc@umass.edu
}

Phone: 413-545-3386; Fax: 413- 545-4490 

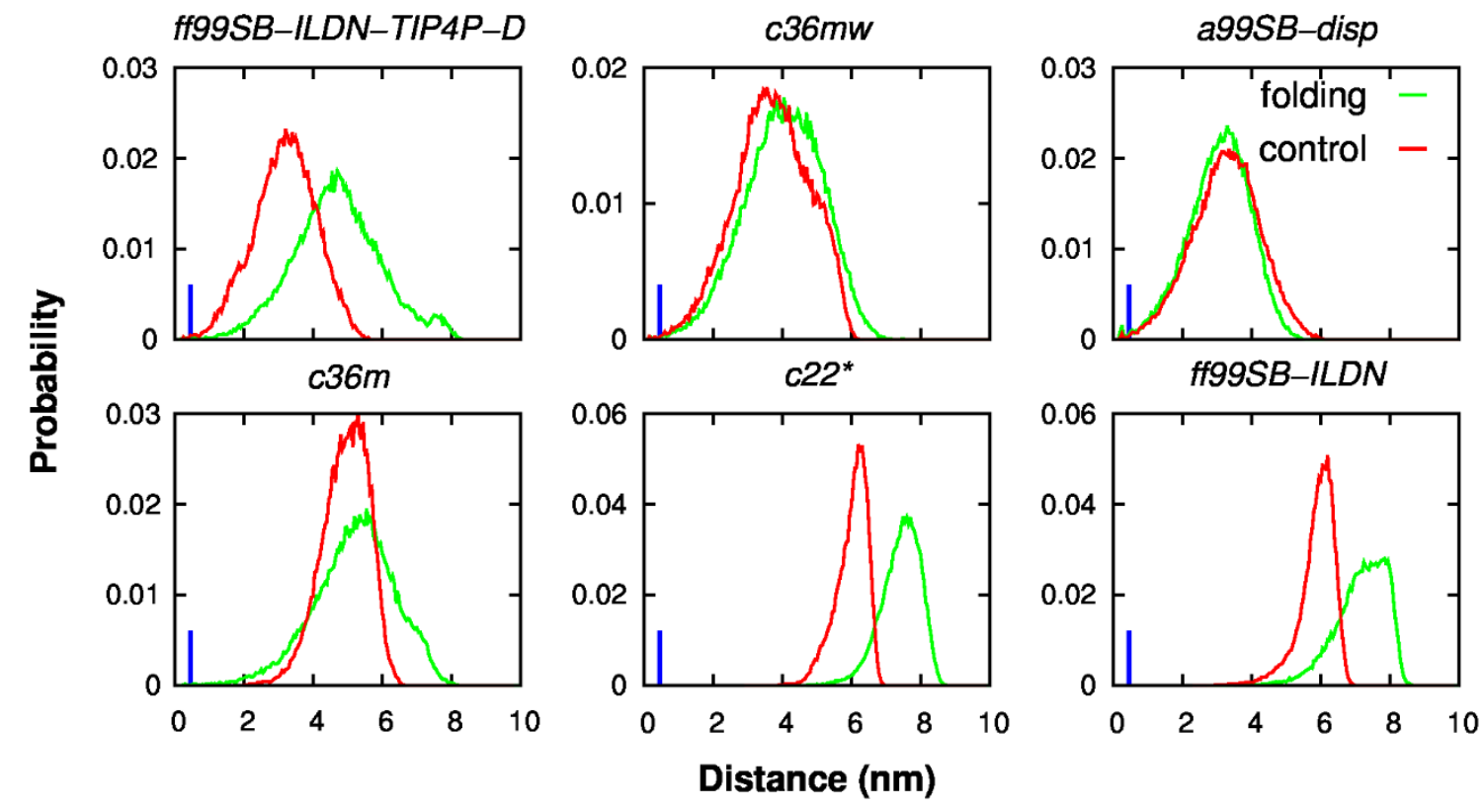

Figure S1. Probability distributions of the minimal distance of p53-TAD to its periodic images. The blue vertical bars indicate $0.45 \mathrm{~nm}$, the cutoff distance for determining if a contact exists. 


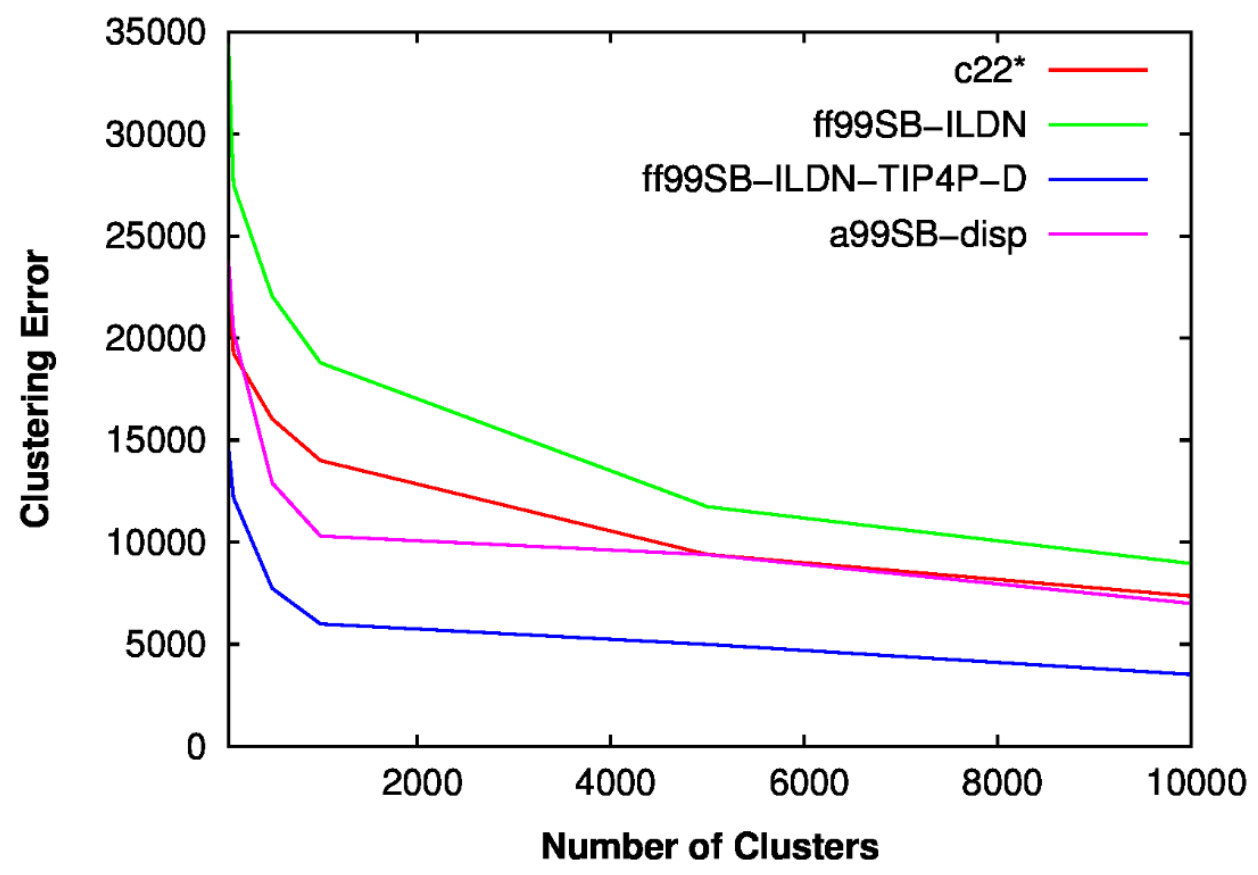

Figure S2. Clustering error (i.e., sum of squared distances of samples to their closest cluster center) as a function of number of clusters for the structural ensembles obtained using four force fields. 

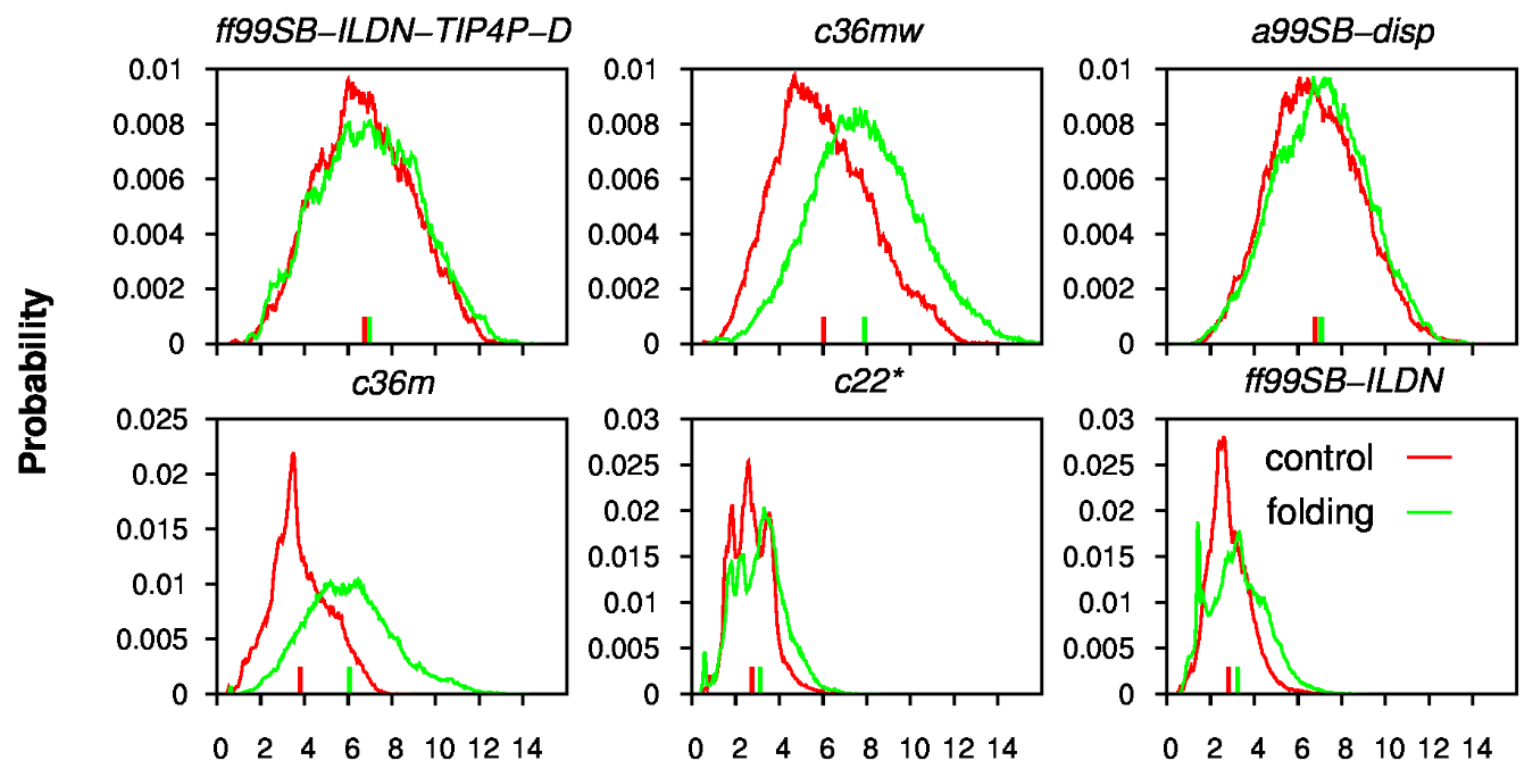

End-to-end Distance (nm)

Figure S3. Probability distributions of the p53-TAD end-to-end distance calculated from independent control (red lines) and folding (green lines) simulations using six force fields. The corresponding ensemble averaged values are indicated using vertical bars. 


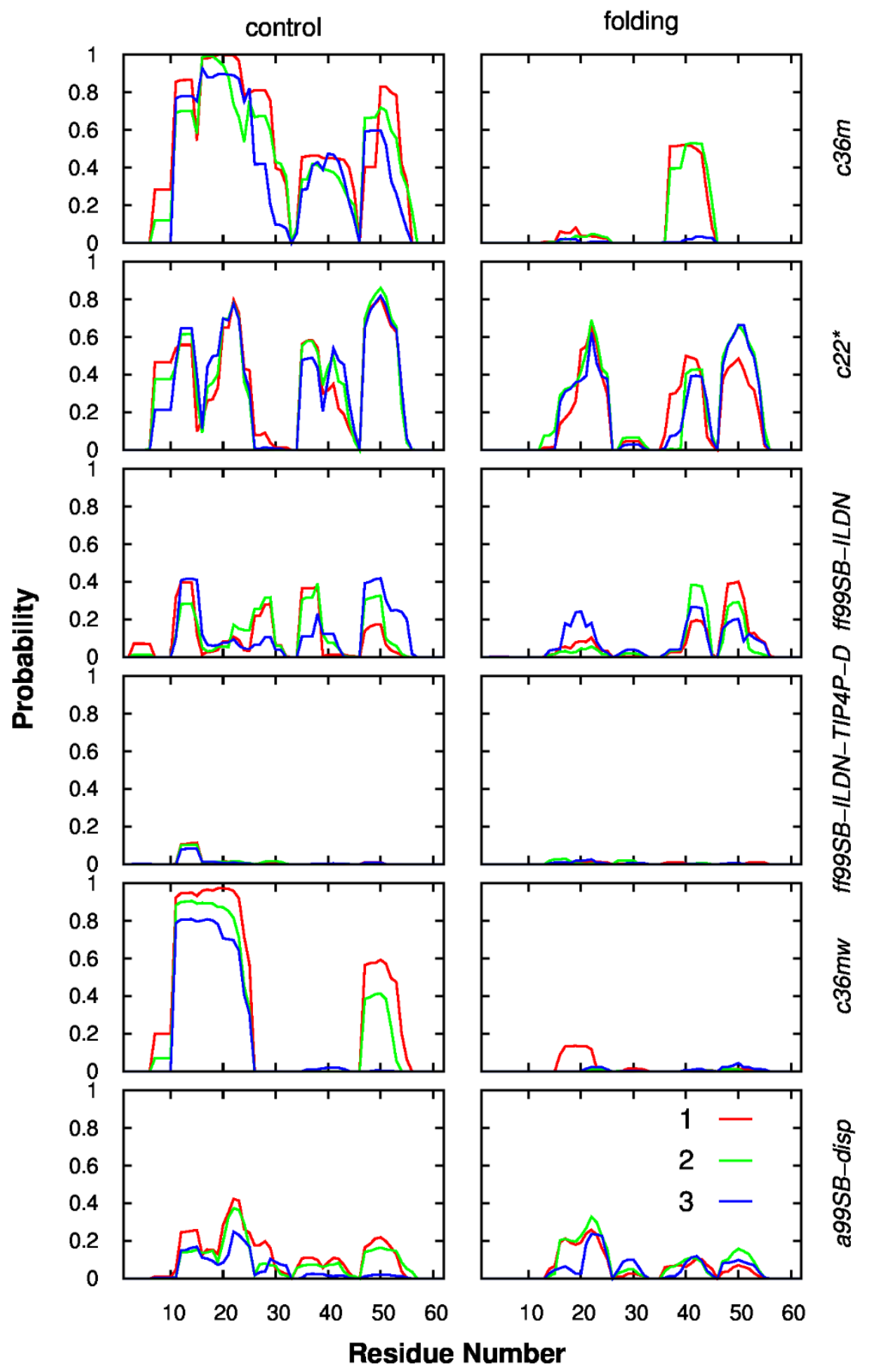

Figure S4. Averaged residue helicity profiles of p53-TAD calculated from the equally divided three portions of each trajectory using six force fields. 


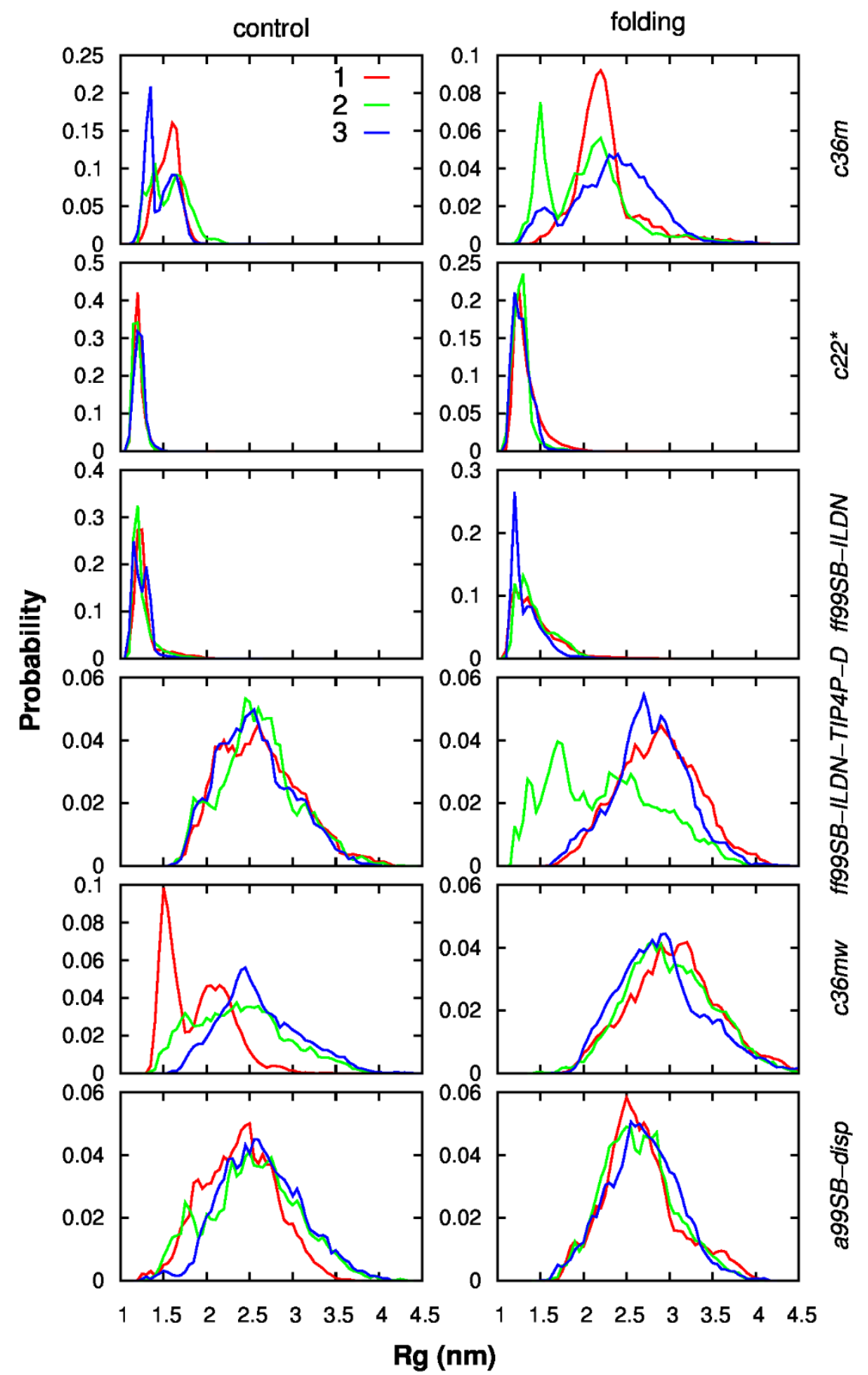

Figure S5. Probability distributions of $R_{\mathrm{g}}$ of p53-TAD calculated from the equally divided three portions of each trajectory using six force fields. 


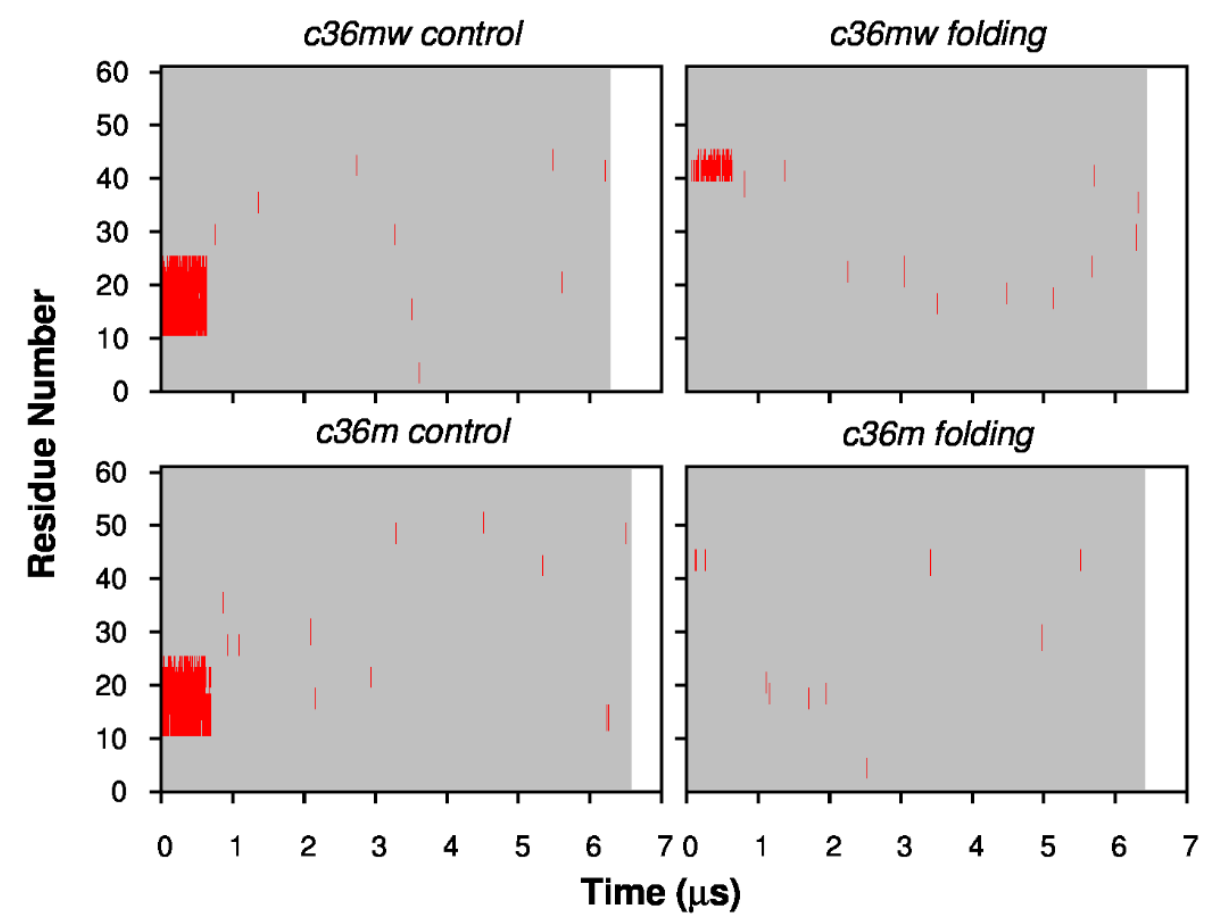

Figure S6. Evolution of the secondary structure of each residue in p53-TAD during standard MD simulations at $298 \mathrm{~K}$. Red indicates helical state, and grey indicates non-helical state. 


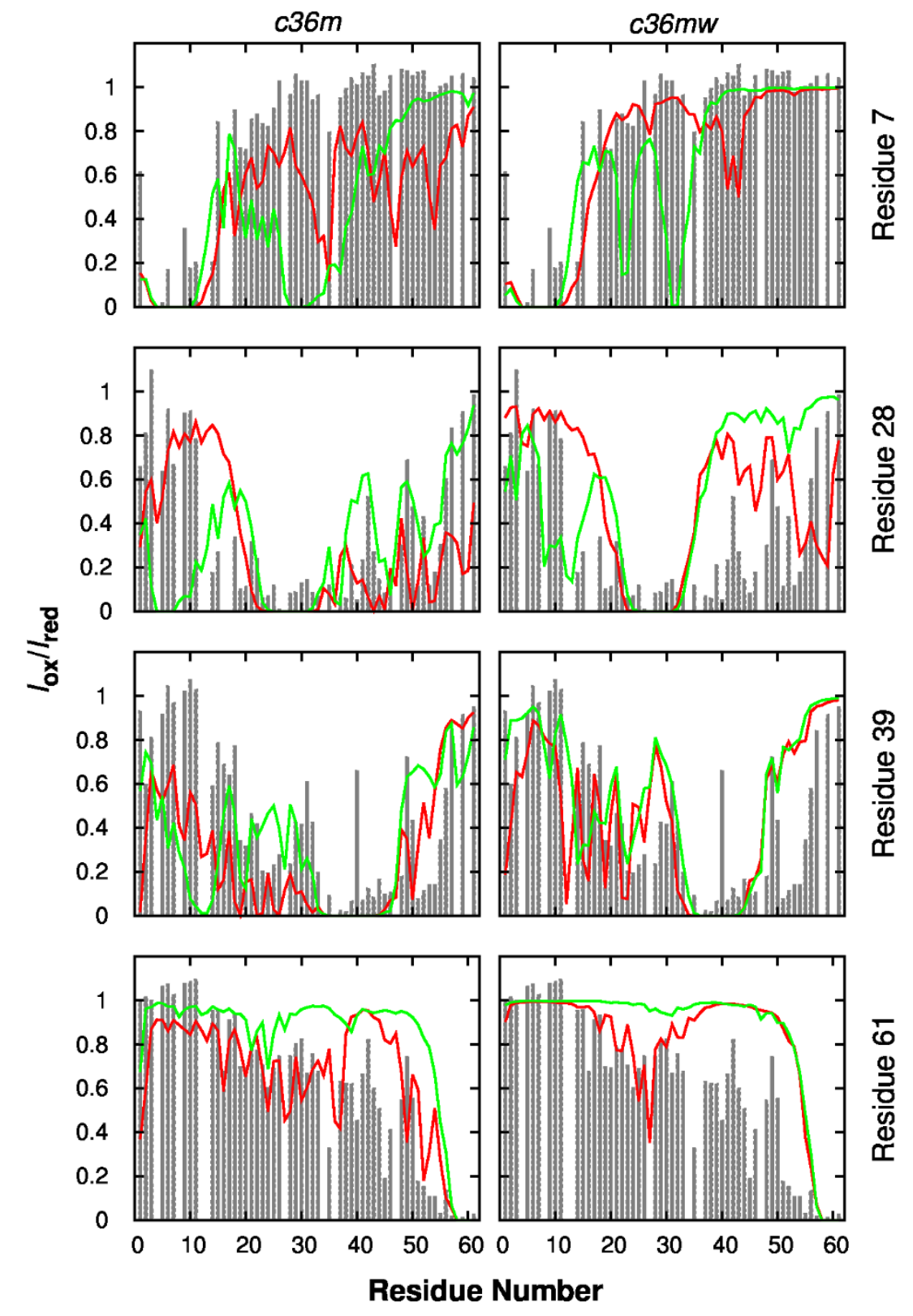

Figure S7. Calculated (lines) and experimental (grey bars) PRE effects induced by paramagnetic spin labelling at residues 7, 28, 39, and 61. Red and green traces are calculated from independent control and folding simulations, respectively, using c36m and c36mw force fields. 


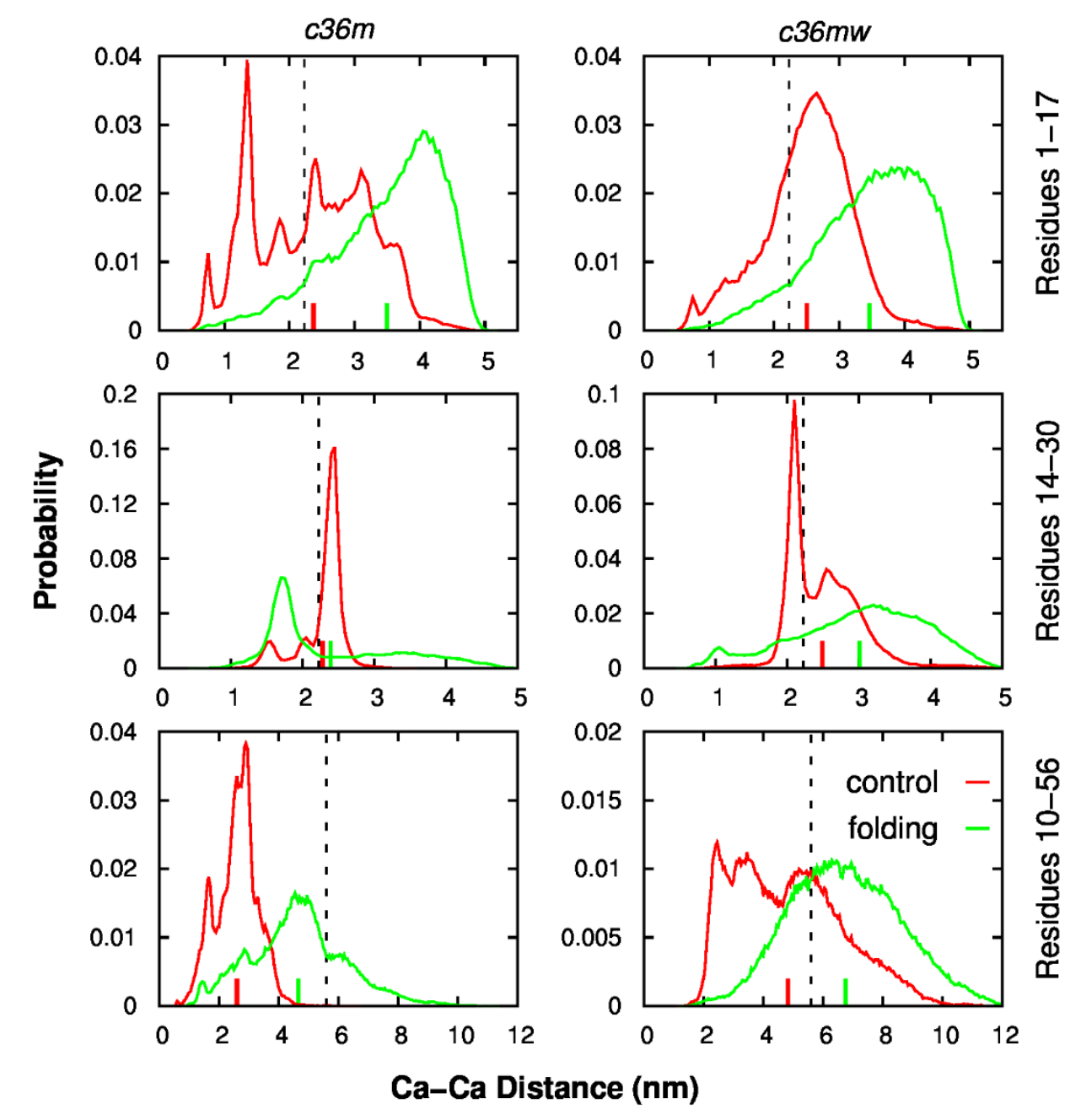

Figure S8. Probability distributions of distances between three pairs of residues, 1 and 17 (top row), 14 and 30 (middle row), and 10 and 56 (bottom row), calculated from independent control (red) and folding (green) simulations using c36m and c36mw force fields. The corresponding ensemble averages were indicated as vertical bars. The vertical black line indicated experimental values from smFRET and TR-FRET measurements. 


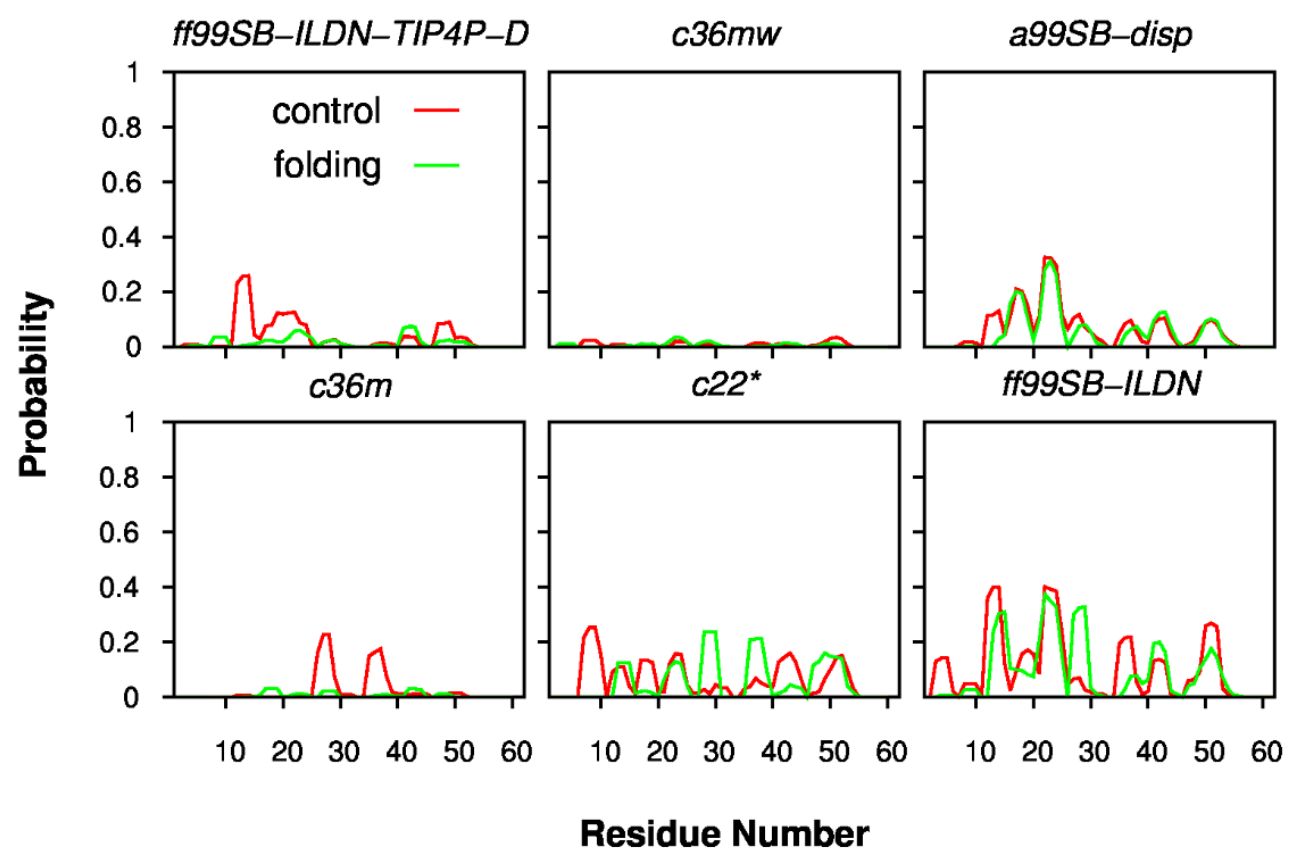

Figure S9. Probability of forming $3_{10}$ helices for each residue of p53-TAD calculated from independent control (red) and folding (green) simulations using six force fields. 


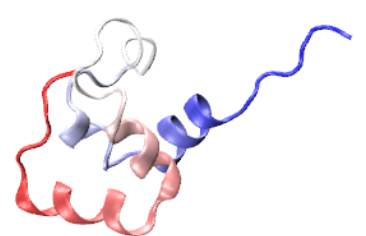

1 (1.56\%)

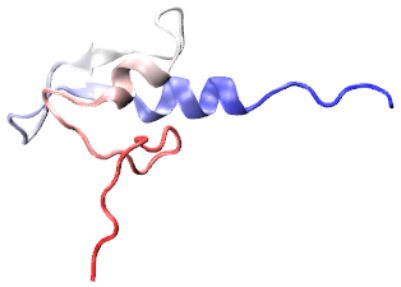

$5(0.97 \%)$

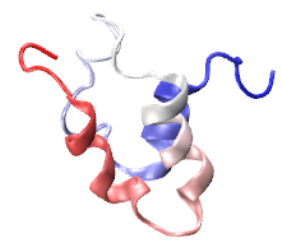

$9(0.87 \%)$

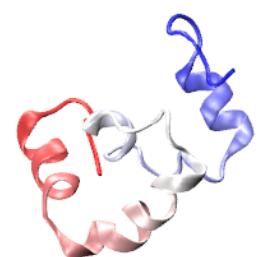

2 (1.54\%)

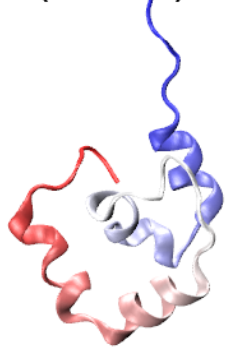

$6(0.95 \%)$

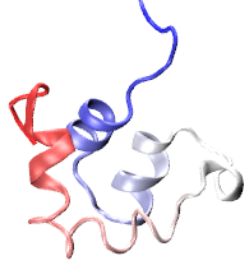

$10(0.83 \%)$

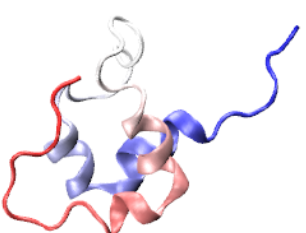

$3(1.52 \%)$

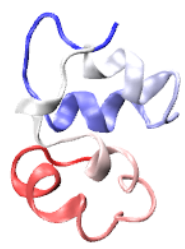

7 (0.90\%)

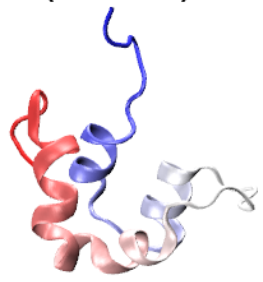

$11(0.83 \%)$

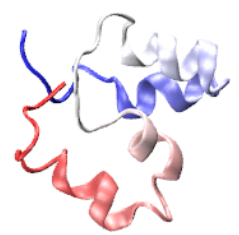

$4(1.10 \%)$

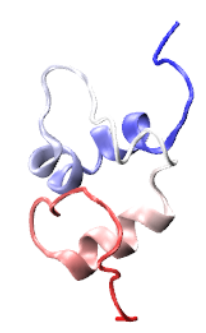

$8(0.87 \%)$

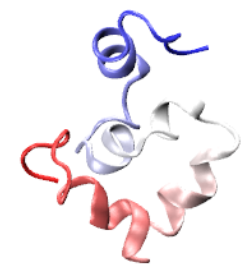

$12(0.82 \%)$

Figure S10. Center structures and populations of top 12 most populated clusters for the structural ensemble derived from $\mathrm{c} 22 *$ simulations. The p53-TAD peptide is shown in Cartoon, with the color changing from red (at $\mathrm{N}$-terminus) to blue (at $\mathrm{C}$-terminus). 


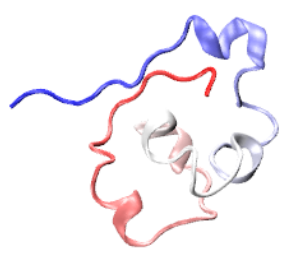

1 (1.21\%)

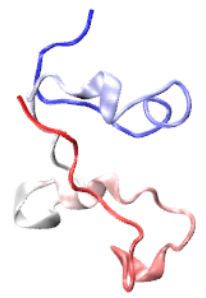

$5(0.80 \%)$

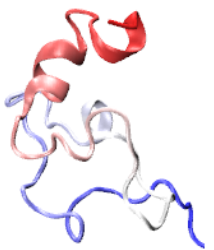

9 (0.69\%)

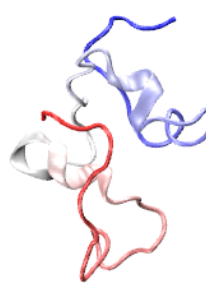

$2(1.07 \%)$
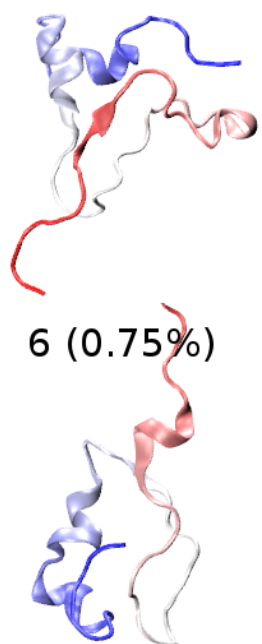

$10(0.68 \%)$

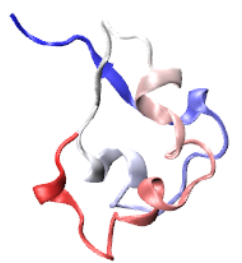

3 (0.94\%)

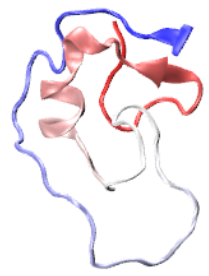

7 (0.73\%)

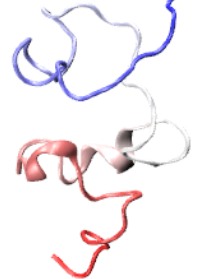

11 (0.66\%)

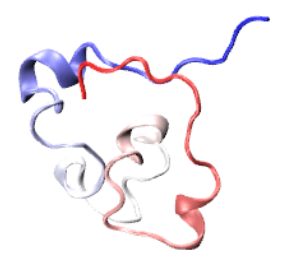

4 (0.89\%)

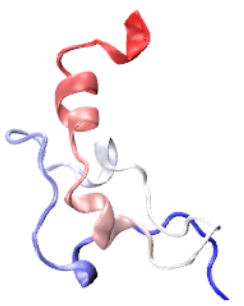

$8(0.73 \%)$

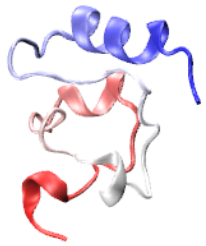

$12(0.65 \%)$

Figure S11. Center structures and populations of top 12 most populated clusters for the structural ensemble derived from ff99SB-ILDN simulations. The p53-TAD peptide is shown in Cartoon, with the color changing from red (at $\mathrm{N}$-terminus) to blue (at C-terminus). 


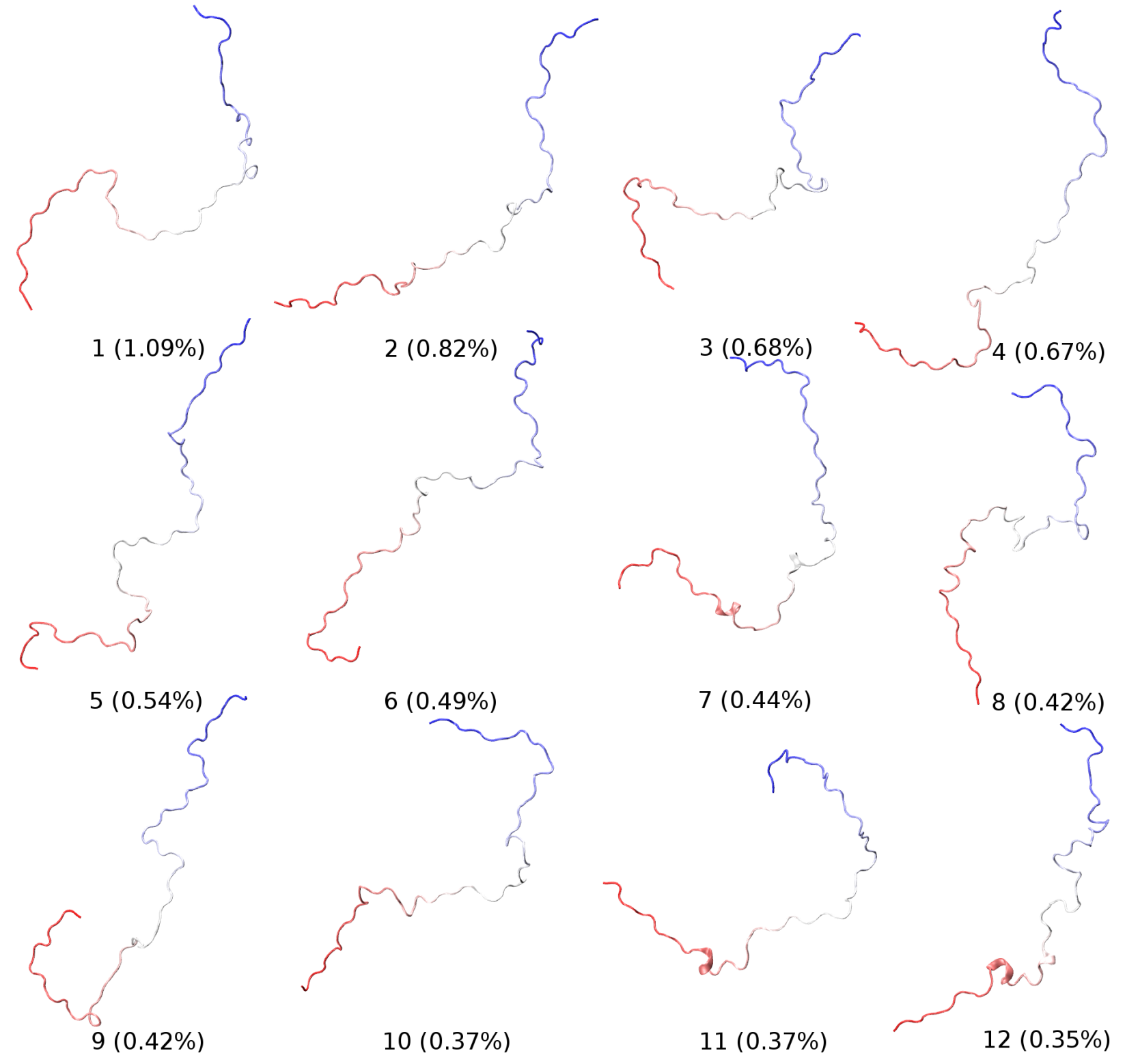

Figure S12. Center structures and populations of top 12 most populated clusters for the structural ensemble derived from ff99SB-ILDN-TIP4P-D simulations. The p53-TAD peptide is shown in Cartoon, with the color changing from red (at N-terminus) to blue (at C-terminus). 